\title{
Isotopic analysis of lead in sub-nanogram quantities by TIMS using a ${ }^{202} \mathrm{~Pb}-{ }^{205} \mathrm{~Pb}$ spike $\dagger$
}

\author{
Y. Amelin* and W. J. Davis \\ Received 15th May 2006, Accepted 25th July 2006 \\ First published as an Advance Article on the web 14th August 2006 \\ DOI: $10.1039 / \mathbf{b 6 0 6 8 4 2 a}$
}

We present a method for isotopic analyses of $\mathrm{Pb}$ in sub-nanogram quantities by thermal ionization mass spectrometry with fractionation correction using a ${ }^{202} \mathrm{~Pb}-{ }^{205} \mathrm{~Pb}$ spike. The procedure is simple, fast, easy to automate, and requires only one analysis of $\mathrm{Pb}$ per sample to acquire both fractionation-corrected $\mathrm{Pb}$ isotopic ratios and $\mathrm{Pb}$ concentration. The procedure is evaluated by analysis of multiple loads of NIST Pb isotopic standards SRM-981 and SRM-982, and $\mathrm{Pb}$ separated from USGS standard rock BCR-1 and chondrite Bruderheim, with the amount of $\mathrm{Pb}$ per load between $0.05-3.0 \times 10^{-9} \mathrm{~g}$. The data have been corrected using both the double spike (DS) fractionation correction described here, as well as by conventional external normalization (EN) procedures, thus providing a direct comparison between the correction methods on exactly the same analytical runs. The enhancement in precision and reproducibility achieved by $\mathrm{DS}$ normalization varies with the quantity of $\mathrm{Pb}$ analysed. For amounts of $\mathrm{Pb}$ of less than $0.2 \mathrm{ng}$, the improvement in precision and reproducibility of ${ }^{207} \mathrm{~Pb} /{ }^{206} \mathrm{~Pb}$ ratios is small but becomes more substantial with increasing sample size: 3-10 times lower within-run errors, and 2-3 times better reproducibility are obtained for $0.5-3.0 \mathrm{ng}$ loads. Even greater enhancement in precision and reproducibility is achieved for the ${ }^{208} \mathrm{~Pb} /{ }^{206} \mathrm{~Pb}$ ratios, at twice the relative mass difference between isotopes. The advantage of DS for the ${ }^{204} \mathrm{~Pb} /{ }^{206} \mathrm{~Pb}$ ratio is, however, much smaller. This indicates that factors other than fractionation control precision and accuracy of isotopic ratios measured at the ion beam levels below $2-3 \times 10^{-13} \mathrm{~A}$-the intensity typically observed for ${ }^{204} \mathrm{~Pb}$ in all samples smaller than $2-3 \mathrm{ng}$, and for all $\mathrm{Pb}$ isotopes in samples smaller than ca. $0.1 \mathrm{ng}$. The method described here is applicable to high-precision $\mathrm{Pb}-\mathrm{Pb}$ and $\mathrm{U}-\mathrm{Pb}$ dating of milligram quantities of meteorites and their components, and single, microgram-size grains of zircon and other U-bearing minerals. The technique described here had been used for dating early solar system materials $(\mathrm{Ca}-\mathrm{Al}$ rich inclusions and primitive achondrites) containing $0.01-0.3 \mathrm{ng}$ of $\mathrm{Pb}$ per fraction, and yielded $\mathrm{Pb}-\mathrm{Pb}$ isochrons with the age error of $0.16-0.33$ million years, which was previously unattainable for such small samples.

\section{Introduction}

The U-Pb method is the most precise, accurate and widely used long-lived isotope chronometer for the determination of the age of rocks and meteorites. In order to obtain precise age information the $\mathrm{U}-\mathrm{Pb}$ method requires high precision measurement of the ${ }^{207} \mathrm{~Pb} /{ }^{206} \mathrm{~Pb}$ ratio. An additional challenge is that high precision must be achieved in analyses of very small quantities of $\mathrm{Pb}(<\mathrm{ng})$ in order to work on well characterized sample materials that contain simple and well preserved $\mathrm{U}-\mathrm{Pb}$ systematics.

Three main factors limit the precision of $\mathrm{Pb}$ isotopic analyses at the sub-nanogram level: (1) uncertainty in the blank correction; (2) instrumental sensitivity and ion detection sys-

Geological Survey of Canada, 601 Booth St., Ottawa, ON, Canada. E-mail: yamelin@nrcan.gc.ca; Fax: (613)995 7997; Tel: (613) 995 3471

$\dagger$ Electronic supplementary information (ESI) available: Ion yields, lead isotopic data, reproducibility information, weighted average isotopic data, accuracy comparison and precision limits. See DOI: $10.1039 / \mathrm{b} 606842 \mathrm{a}$ tems (counting statistics, signal to noise ratio; multiplier corrections for dead time and non-linearity); and (3) accurate and precise correction for instrumental mass fractionation during analyses. The first of these can be largely obviated (at least for the samples containing more than $c a .0 .1 \mathrm{ng} \mathrm{Pb}$ ) through low-blank clean lab procedures that permit procedural blanks of less than $1 \mathrm{pg}$. Thermal ionization mass spectrometry (TIMS) is the most sensitive technique for isotopic analysis of lead separated from rocks and minerals, with ion yields (the ratio of the number of ions registered, to the number of atoms of the same nuclide in the sample processed for analysis) up to $c a .10 \% .{ }^{1}$ For comparison, the ion yield for $\mathrm{Pb}$ in state-of-the-art MC-ICP-MS with the use of desolvating nebulizers for maximum sensitivity is between $0.39-1.72 \%$ (calculated from the data given in refs. 2-5, details in the Electronic Supplementary Information Table $\mathrm{S} 1 \dagger)$. Additional advantages of TIMS over MC-ICP-MS are very low background and the absence of memory. The precision and accuracy of $\mathrm{Pb}$ isotopic analysis by TIMS is, however, limited by uncertainties in applying mass fractionation correction, a factor that varies from load to load, as well as over the course 
of a single analysis as the sample evaporates from the filament. Mass fractionation cannot be corrected using internal normalization, because natural $\mathrm{Pb}$ does not have a pair of isotopes with a constant abundance ratio. Typical uncertainties due to fractionation correction using external normalization procedures are $\pm 0.03 \%$ to $\pm 0.05 \%$ per atomic mass unit, and represent a very significant source of uncertainty in $\mathrm{Pb}$ analyses by TIMS.

The precision, accuracy and reproducibility of $\mathrm{Pb}$ isotope analysis can be improved by using a double (or triple) spike (DS) to correct instrumental mass fractionation. ${ }^{6-10}$ The ${ }^{204} \mathrm{~Pb}-{ }^{207} \mathrm{~Pb}$ DS has been used for high-precision isotopic analyses of large amounts of $\mathrm{Pb}$ from terrestrial rocks for almost 40 years. ${ }^{11-13}$ This method is, however, poorly suited to the analysis of sub-nanogram amounts of radiogenic $\mathrm{Pb}$ because the samples must be aliquotted for spiking and this can introduce additional error due to non-uniformity of the procedural blank, and requires even greater instrumental sensitivity as sample utilization is less efficient. In addition, the $\mathrm{Pb}$ isotopic composition of the unspiked aliquot is extremely sensitive to potential accidental contamination with ${ }^{204} \mathrm{~Pb}-{ }^{207} \mathrm{~Pb}$ spike. A DS made of artificial isotopes ${ }^{202} \mathrm{~Pb}$ and ${ }^{205} \mathrm{~Pb}^{14}$ is free from these problems. A procedure with the use of mixed ${ }^{202} \mathrm{~Pb}-{ }^{205} \mathrm{~Pb}$ spike requires only one analysis per sample, from which both fractionation-corrected isotopic ratios and precise concentration of $\mathrm{Pb}$ (from fractionationcorrected sample/spike ratio) are determined.

Here we present a simple technique for the measurement of the isotopic composition of $\mathrm{Pb}$ in nanogram and sub-nanogram quantities with fractionation correction using a ${ }^{202} \mathrm{~Pb}-{ }^{205} \mathrm{~Pb} \mathrm{DS}$. This technique has been tested by analyses of 0.05-3 ng of $\mathrm{Pb}$ from National Institute of Standards and Technology (NIST) isotopic standards and rock standardsthe approximate amount of $\mathrm{Pb}$ in milligram-sized meteorite samples and in single grains of zircon. We explore the lower sample size limit of efficient use of the DS by comparing the precision and reproducibility of the same $\mathrm{Pb}$ isotopic data reduced with DS and conventional external normalization (EN) over a range of sample sizes.

\section{Experimental}

\subsection{Preparation and isotopic composition of ${ }^{202} \mathrm{~Pb}-{ }^{205} \mathrm{~Pb}$ DS}

The ${ }^{202} \mathrm{~Pb}-{ }^{205} \mathrm{~Pb}$ mixed spike was prepared and calibrated at the Geological Survey of Canada from high-purity ${ }^{202} \mathrm{~Pb}$ provided by $\mathrm{W}$. Todt ${ }^{14}$ and ${ }^{205} \mathrm{~Pb}$ provided by G. J. Wasserburg ${ }^{15}$ (about $10^{-6} \mathrm{~g}$ of ${ }^{202} \mathrm{~Pb}$ and ${ }^{205} \mathrm{~Pb}$ each). A half of the ${ }^{202} \mathrm{~Pb}-{ }^{205} \mathrm{~Pb}$ spike was mixed with the ${ }^{233} \mathrm{U}-{ }^{235} \mathrm{U}$ double spike ${ }^{16}$ currently in use at the GSC. The ${ }^{202} \mathrm{~Pb} /{ }^{205} \mathrm{~Pb}$ ratio of $1.020032 \pm 0.000009$ is calibrated against ${ }^{208} \mathrm{~Pb} /{ }^{206} \mathrm{~Pb}=$ 2.1677 in the SRM-981 standard (the weighted average from seven runs of 10-100 ng loads of SRM-981 mixed with the ${ }^{202} \mathrm{~Pb}-{ }^{205} \mathrm{~Pb}$ spike). The abundances of other isotopes $\left({ }^{204} \mathrm{~Pb} /{ }^{205} \mathrm{~Pb}=1.09 \times 10^{-4},{ }^{206} \mathrm{~Pb} /{ }^{205} \mathrm{~Pb}=9.91 \times 10^{-4}\right.$, ${ }^{207} \mathrm{~Pb} /{ }^{205} \mathrm{~Pb}=8.46 \times 10^{-4},{ }^{208} \mathrm{~Pb} /{ }^{205} \mathrm{~Pb}=2.054 \times 10^{-3}$ ) are sufficiently low to make uncertainty in the spike subtraction insignificant for all but the most severely overspiked samples

(the additional uncertainty in ${ }^{207} \mathrm{~Pb} /{ }^{206} \mathrm{~Pb}$ due to spike subtraction is less than $0.01 \%$ if ${ }^{205} \mathrm{~Pb} /{ }^{206} \mathrm{~Pb}<10$ ).

\subsection{Chemical separation, reagents, and blanks}

$\mathrm{Pb}$ was separated from rock samples by double-pass-through columns containing $50 \mu \mathrm{l}$ of anionite AG50Wx8, using the procedure described in refs. 17 and 18. We used Baselinebrand $\mathrm{HBr}, \mathrm{HCl}, \mathrm{HF}$ and $\mathrm{HNO}_{3}$ (from Seastar Chemicals) without further purification, and high-purity water from a Milli-Q system (Millipore). All reagents have $\mathrm{Pb}$ concentrations well below 1 ppt. The total procedure blanks were measured with each batch of samples (usually 2-3 blanks per batch), and were typically between 1-3 pg. Blank correction, with appropriate error magnification, ${ }^{19}$ was done for all samples, using the average $\mathrm{Pb}$ isotopic composition of the blank measured with each batch.

The filament loading blanks were between $0.3-0.4 \mathrm{pg}$ in the early stages of the study. The loading blank dropped to the average value of $0.08 \mathrm{pg}$ after replacement of a poor-quality dispensing bottle used for $0.03 \mathrm{M} \mathrm{H} \mathrm{H}_{3} \mathrm{PO}_{4}$. Analyses of all standards loaded without chemical separation were corrected for a representative loading blank.

Standards were spiked with ${ }^{202} \mathrm{~Pb}-{ }^{205} \mathrm{~Pb}$ tracer containing between 10 and $300 \mathrm{pg}$ of ${ }^{205} \mathrm{~Pb}$, and the standard-spike mixture was evaporated with $0.01 \mathrm{ml}$ of $0.03 \mathrm{M} \mathrm{H}_{3} \mathrm{PO}_{4}$ in a Teflon PFA vial (Savillex Corp.). Aliquots of sample solutions were spiked in a similar way before chemical separation.

\subsection{Instrumentation and running conditions}

All isotopes between 201 and 208 were measured in Faraday cups in a static multi-collector mode on the Triton TI mass spectrometer at the Geochronology Laboratory, Geological Survey of Canada, under conditions summarized in Table 1.

Each filament was automatically heated to $1150-1200{ }^{\circ} \mathrm{C}$ over 8-10 $\min \left(200 \mathrm{~mA} \mathrm{~min}^{-1}\right)$ during which time the baseline measurements were carried out with the Line of Sight (LOS) closed. The ion beam was focused and $\mathrm{Pb}$ was measured in semi-automatic mode, with an automated temperature increment of $15{ }^{\circ} \mathrm{C}$ after each block of 15 integrations of $8 \mathrm{~s}$. A typical run consisted of 10-20 blocks, and the data were collected in a temperature range of $150-300{ }^{\circ} \mathrm{C}$. Isobaric interferences were monitored on the masses $201\left(\mathrm{BaPO}_{2}{ }^{+}\right)$

Table 1 Typical instrument operating conditions

Instrument

Accelerating voltage

Source pressure

Analyzer pressure

Faraday collector gain

calibration

Amplifier baseline

measurement

Amplifier rotation

Filaments

Emission activator

Filament temperature range

Total duration of analysis

\author{
Triton T1 (Thermo Finnigan) \\ $10000 \mathrm{~V}$ \\ 4-8 $\times 10^{-8}$ mbar \\ (with liquid $\mathrm{N}_{2}$ cold trap) \\ $3 \times 10^{-9}$ mbar \\ Daily \\ 8 min total integration during \\ sample warm-up \\ Disabled \\ Zone-refined rhenium $99.999 \%$ \\ (H. Cross) \\ Colloidal silicic acid (Merck, ref.1) \\ Start $1150-1250{ }^{\circ} \mathrm{C}$, \\ finish $1350-1500{ }^{\circ} \mathrm{C}$ \\ 40-60 min
}


and $203\left(\mathrm{Tl}^{+}\right)$. The $\mathrm{BaPO}_{2}{ }^{+}$ion beam was detected in almost all analyses, and ${ }^{202} \mathrm{~Pb},{ }^{203} \mathrm{~Pb}$ and ${ }^{204} \mathrm{~Pb}$ were therefore corrected for $\mathrm{BaPO}_{2}{ }^{+}$interference. The uncertainty related to this correction is greatly de-magnified by low $202 / 201=1.27 \times$ $10^{-3}$ (average empirical value) and $204 / 201=1.5 \times 10^{-6}$ in $\mathrm{BaPO}_{2}{ }^{+}$. No statistically significant signal was observed on the mass 203 after correction for $\mathrm{BaPO}_{2}{ }^{+}$, thus no correction for $\mathrm{Tl}$ interference was applied to ${ }^{205} \mathrm{~Pb}$. Isotopic ratios corrected for fractionation (assuming an exponential law using on-line software supplied by Finnigan) and uncorrected were collected. Subsequent data reduction was performed off-line. The mean values of isotopic ratios for each run were calculated using the "Weighted average" function of Isoplot Ex 3.00. ${ }^{20}$ Final isotopic ratios corrected for fractionation, blank, and spike, and their errors and error correlations, were calculated using the PBDAT program. ${ }^{21}$

\section{Results}

\subsection{Isotopic data}

Fifty-five spiked loads of the NIST standard SRM-981, containing between 0.053 and $1.914 \mathrm{ng} \mathrm{Pb}$ were analysed, as well as smaller numbers of NIST SRM-982, Alfa Aesar Pb (Purotronic brand, http://earth-time.org/JMM_U $=$ Pb_cal.doc.xls, referred to as Mattinson $\mathrm{Pb}$ ), US Geological Survey standard basalt BCR-1 (position 20, split 56), and a split of powdered meteorite (L6 chondrite) Bruderheim (University of California Berkeley standard, sample Bru-7-8, split 128). BCR-1 and Bruderheim were analyzed in order to test the possible effects of residual sample matrix on $\mathrm{Pb}$ isotopic fractionation. The concentration of $\mathrm{Pb}$ in BCR-1 is $c a .50$ times higher than in Bruderheim, therefore the latter can be expected to have a much higher ratio of residual matrix elements to $\mathrm{Pb}$, assuming similar separation efficiency for mafic (BCR-1) and ultramafic (Bruderheim) compositions. Homogenized solutions (dilute $\mathrm{HCl}$ ) of each rock standard were prepared from a single dissolution of a large quantity ( $c a$. $0.5 \mathrm{~g}$ ), and aliquots of these solutions were separately spiked and processed in this study. This procedure assures that any variations in $\mathrm{Pb}$ isotopic composition between the aliquots are analytical, and not caused by sample heterogeneity. In order to test the possible effect of organics from ion-exchange resin, three $c a$. $0.186 \mathrm{ng}$ loads of SRM-981 Pb were processed with the chemical separation procedure prior to analysis.

The data are summarized in ESI Table S $\dagger \dagger$. The data in the upper half of the table are normalized to ${ }^{202} \mathrm{~Pb} /{ }^{205} \mathrm{~Pb}$ using the exponential fractionation law. Normalization was applied online to every 8 -s integration. The same data, processed with conventional external normalization, are shown in the lower half of the table. For external normalization, the average fractionation value of $0.097 \pm 0.036 \%$ (2 SD) calculated from the entire database of our $\mathrm{Pb} \mathrm{DS}$ measurements acquired so far was used. The error in the external fractionation value was applied quadratically to the weighted mean error of the individual block results and represents a minimum error for externally-calibrated runs.

The first portion of the data for SRM-981 were acquired during setting up the DS procedure in May-July 2005. Addi- tional standards were run over the period of July 2005 to February 2006, along with samples as a part of routine monitoring of the instrument performance. These data therefore represent a long-term reproducibility.

\subsection{Within-run variations}

Detailed data and plots showing individual block values for ion beam intensity and directly measured and normalized isotopic ratios for SRM-981 are presented in ESI Table S2. Representative plots are shown in Fig. 1.

The ion beam intensity typically increases 10-30 times between the beginning of each analysis and the intensity maximum, which in most cases occurred between blocks 10 and 15 . The intensity versus temperature (or analysis time, expressed by the block number) pattern varies between the loads, but does show regular variations with sample size. The measured ratios of heavier isotopes to lighter isotopes, represented here by ${ }^{207} \mathrm{~Pb} /{ }^{206} \mathrm{~Pb}$, increase, as expected, during the run, because of increasing sample depletion in more volatile lighter isotopes. ${ }^{22}$ For small samples (e.g., $30 \mathrm{~A}$ on Fig. 1), the change in isotopic ratios due to fractionation is comparable to the errors of individual data blocks, therefore normalization results in only a marginal improvement of within-run precision. For the larger samples, the block errors are much smaller but the fractionation-related drift is the same as for small samples. Accordingly, correction of fractionation yields much greater improvement in precision.

The precision of isotopic ratios obtained with DS and EN fractionation correction are compared in Fig. 2. In all cases investigated the DS correction yields more precise results than the EN method. The improvement is most evident for larger sample loads ( $>1 \mathrm{ng}$ ) in which the DS normalized results are 3-10 times more precise than EN normalized data. For smaller sample loads between $0.05-0.19 \mathrm{ng}$ the improvement is less than a factor of two.

\subsection{Reproducibility}

The reproducibility of SRM-981 analyses reduced with DS is illustrated in Fig. 3. The average values and reproducibility for all samples and standards is summarized in Table 2.

For the smallest $(0.057 \mathrm{ng})$ loads, the reproducibility of DSnormalized and EN-normalized ${ }^{207} \mathrm{~Pb} /{ }^{206} \mathrm{~Pb}$ and ${ }^{208} \mathrm{~Pb} /{ }^{206} \mathrm{~Pb}$ ratios is similar. For the ${ }^{207} \mathrm{~Pb} /{ }^{206} \mathrm{~Pb}$ in larger samples, DS improves reproducibility by $c a$. 1.5 times $(0.032 \%$ versus $0.047 \%)$ for $0.185 \mathrm{ng}$ loads, 1.8 times $(0.016 \%$ versus $0.029 \%$ ) for $0.564 \mathrm{ng}$ loads, and 2.4 times $(0.007 \%$ versus $0.017 \%$ ) for $1.874 \mathrm{ng}$ loads of SRM-981. Even greater improvement, up to three times, is observed for the rock samples. The improvement of reproducibility due to using DS is greater for ${ }^{208} \mathrm{~Pb} /{ }^{206} \mathrm{~Pb}$ than for ${ }^{207} \mathrm{~Pb} /{ }^{206} \mathrm{~Pb}$, probably because of the larger mass difference. $\mathrm{The}{ }^{204} \mathrm{~Pb} /{ }^{206} \mathrm{~Pb}$ ratio, on the contrary, shows only marginal, if any, improvement in precision, over the entire range of sample sizes. This indicates that factors other than fractionation determine the precision and accuracy of isotopic ratios measured at the ion beam levels below 2-3 $\times$ $10^{-13} \mathrm{~A}$ - the intensity typically observed for ${ }^{204} \mathrm{~Pb}$ in all samples smaller than 2-3 ng, and for all $\mathrm{Pb}$ isotopes in samples smaller than ca. $0.1 \mathrm{ng}$. 

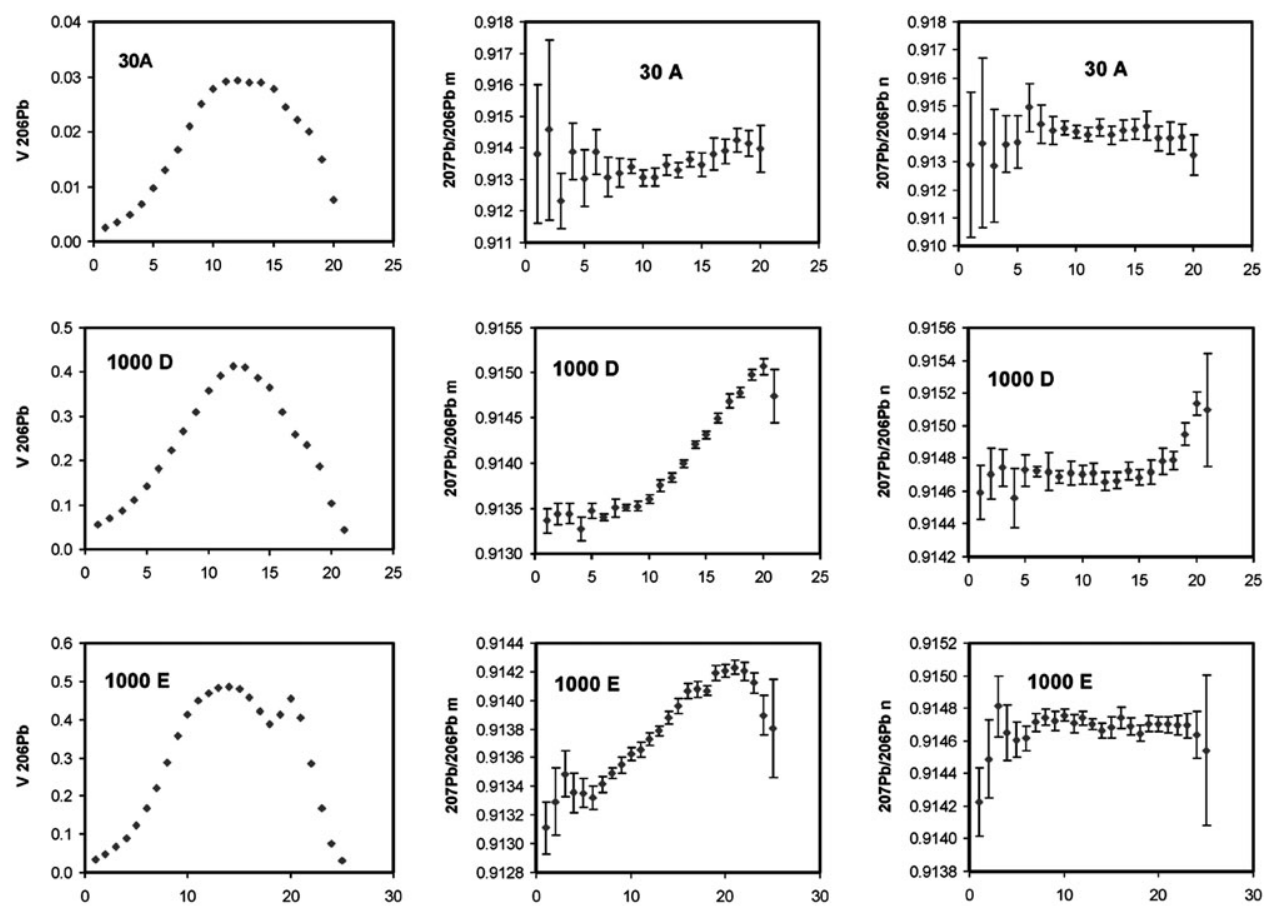

Fig. 1 Within-run variations of the ion beam intensity (left column), measured ${ }^{207} \mathrm{~Pb} /{ }^{206} \mathrm{~Pb}$ (middle column), and normalized ${ }^{207} \mathrm{~Pb} /{ }^{206} \mathrm{~Pb}($ right column) in representative small loads (30 A, $0.058 \mathrm{ng}$ ) and large loads (1000 D and $1000 \mathrm{E}, 1.865 \mathrm{ng})$ of SRM-981.

Comparison of the data in Figs. 2 and 3 shows that using DS causes greater improvement in within-run precision than in long-time reproducibility. The reproducibility is thus limited, to some extent, by factors other than fractionation uncertainty. Achieving the highest possible within-run precision is

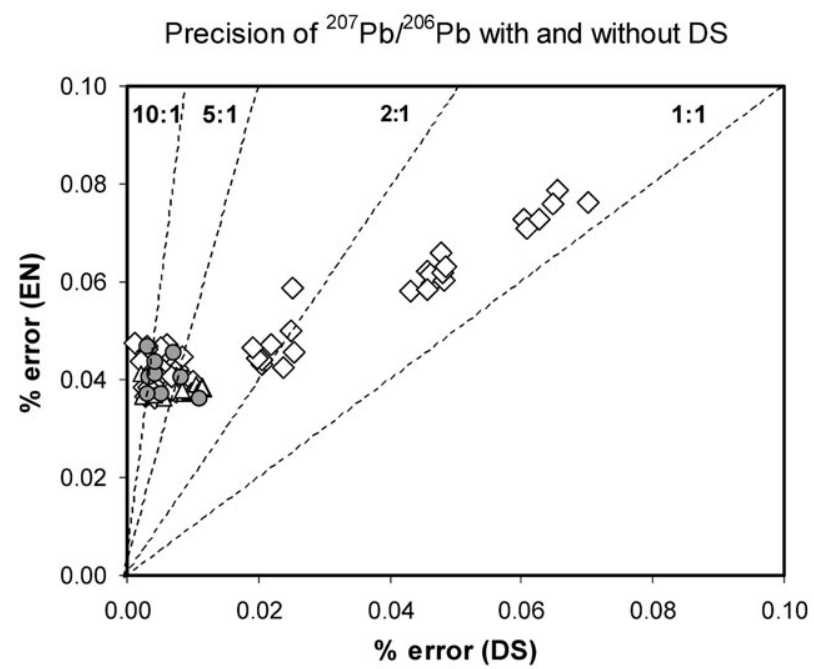

Fig. 2 The relationship of within-run errors (relative 95\% errors of weighted average) for $\mathrm{Pb}$ isotopic ratios corrected for fractionation using double spike (DS) and external normalization (EN) procedures. SRM-981standard - open diamonds, spike calibration mixturesopen triangles, rock samples - closed circles. Diagonal broken lines show constant ratios of EN error to DS error between 1:1 and 10:1. Four clusters of data points, from right to left, represent loads of $57 \mathrm{pg}, 185 \mathrm{pg}, 564 \mathrm{pg}$ and $>1000 \mathrm{pg}$, respectively. necessary, however, for recognizing and eliminating these factors.

\subsection{Accuracy}

The weighted mean values of DS-corrected isotopic ratios are calculated in ESI Table S4 $\uparrow$, and compared with the published values for SRM-981, SRM-982 and BCR-1 in Table S5†. Although this study was not designed to determine the most accurate standard values, the values for SRM-981 and SRM-

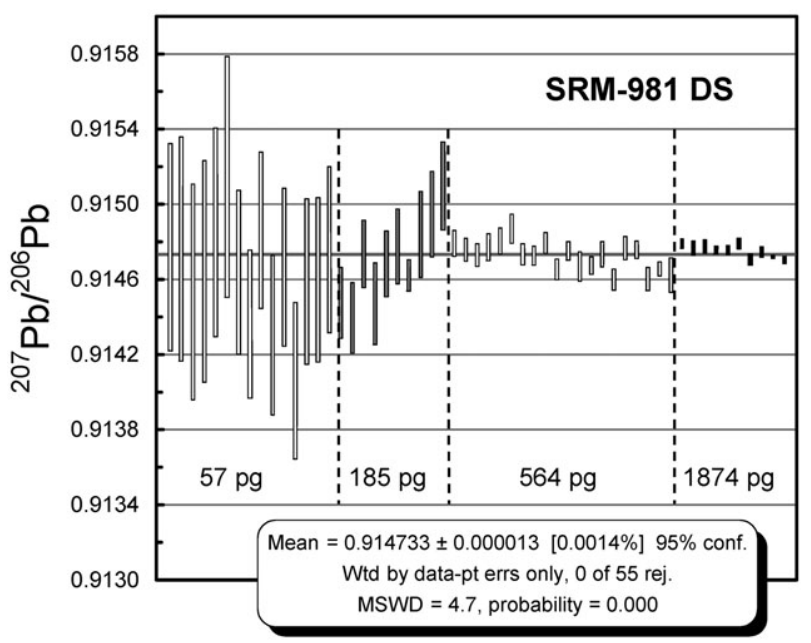

Fig. 3 Reproducibility of SRM-981 analyses reduced with DS (internal normalization to ${ }^{202} \mathrm{~Pb} /{ }^{205} \mathrm{~Pb}$ ). Number at the bottom of the graph indicates the average amount of $\mathrm{Pb}$ in the loads of each cluster. Actual sizes of loads vary within $3-5 \%$ due to the uncertainty in the measurements of the amounts of sample and spike solutions. 
Table 2 Reproducibility of replicate analyses of $\mathrm{Pb}$ standards and samples

\begin{tabular}{|c|c|c|c|c|c|c|c|c|c|c|c|c|c|}
\hline Sample name & $\begin{array}{l}F(202 / \\
205)\end{array}$ & $\begin{array}{l}2 \sigma \% \\
\text { error }\end{array}$ & $\begin{array}{l}\text { Ion } \\
\text { yield } \\
(\%)\end{array}$ & $\begin{array}{l}2 \sigma \% \\
\text { error }\end{array}$ & $\mathrm{Pb}(\mathrm{ppm})$ & $\mathrm{Pb} / \mathrm{pg}$ & $\begin{array}{l}2 \sigma \% \\
\text { error }\end{array}$ & $\begin{array}{l}{ }^{204} \mathrm{~Pb} / \\
{ }^{206} \mathrm{~Pb}\end{array}$ & $\begin{array}{l}2 \sigma \% \\
\text { error }\end{array}$ & $\begin{array}{l}{ }^{207} \mathrm{~Pb} / \\
{ }^{206} \mathrm{~Pb}\end{array}$ & $\begin{array}{l}2 \sigma \% \\
\text { error }\end{array}$ & $\begin{array}{l}{ }^{208} \mathrm{~Pb} / \\
{ }^{206} \mathrm{~Pb}\end{array}$ & $\begin{array}{l}2 \sigma \% \\
\text { error }\end{array}$ \\
\hline \multicolumn{14}{|c|}{ Double spike normalization } \\
\hline SpikeCal SRM N982 & 1.00128 & .019 & 1.8 & 73 & 0.602 & 1885 & 25 & 0.02721 & 0.131 & 0.46702 & 0.011 & 1.00004 & 0.014 \\
\hline SpikeCal Mattinson & 1.00115 & 0.034 & 1.9 & 43 & 0.115 & 2043 & 5 & 0.05490 & 0.030 & 0.85676 & 0.007 & 2.10189 & 0.003 \\
\hline SRM-981 57 pg & 1.00091 & 0.031 & 6.7 & 49 & 0.002 & 57 & 10 & 0.05903 & 0.397 & 0.91464 & 0.057 & 2.16768 & 0.067 \\
\hline SRM-981 185 pg & 1.00098 & 0.034 & 6.8 & 45 & 0.002 & 185 & 2 & 0.05902 & 0.173 & 0.91459 & 0.032 & 2.16756 & 0.028 \\
\hline SRM-981 185 pg proc. & 1.00086 & 0.035 & 5.9 & 64 & 0.002 & 186 & 0.4 & 0.05905 & 0.098 & 0.91496 & 0.029 & 2.16804 & 0.017 \\
\hline $1564 \mathrm{pg}$ & 1.00093 & 0.021 & 5.2 & 63 & 0.002 & 564 & 4 & 0.05901 & 0. & 0.9 & 16 & 2.1 & 0.019 \\
\hline SRM 9811874 pg & 1.00091 & 0.018 & 3.6 & 51 & 0.002 & 1873 & 2 & 0.05902 & 0.028 & 0.91475 & 0.007 & 2.16778 & 0.013 \\
\hline Bruderheim & 1.00096 & 0.030 & 2.8 & 55 & 0.293 & 1467 & 2 & 0.04875 & 0.118 & 0.82396 & 0.012 & 1.96027 & 0.011 \\
\hline BCR-1 & 1.00100 & 0.023 & 3.2 & 128 & 15.571 & 2958 & 4 & 0.05312 & 0.063 & 0.83079 & 0.009 & 2.05797 & 0.018 \\
\hline \multicolumn{14}{|l|}{ External normalization } \\
\hline SpikeCal SRM 982 & 1.00128 & 019 & 1.8 & 73 & 0 & 1884 & 25 & 0.02723 & 0.136 & 0.46690 & 0.018 & 0.99947 & 0.032 \\
\hline SpikeCal Mattinson & 1.00115 & 0.034 & 1.9 & 43 & 0.115 & 2042 & 5 & 0.05491 & 0.072 & 0.85662 & 0.035 & 2.10100 & 0.052 \\
\hline $8157 \mathrm{pg}$ & 1.00091 & 0.031 & 6.7 & 49 & 0.002 & 57 & 10 & 0.05902 & 0.381 & 0.91471 & 0.042 & 2.16806 & 0.064 \\
\hline SRM-981 $185 \mathrm{pg}$ & 1.00098 & .034 & 6.8 & 45 & 0.002 & 185 & 3 & 0.05900 & 0.188 & 0.91467 & 0.047 & 2.16779 & 0.066 \\
\hline SRM-981 185 pg proc. & 1.00086 & 0.035 & 5.9 & 64 & 0.002 & 186 & 0.3 & 0.05904 & 0.140 & 0.91503 & 0.017 & 2.16827 & 0.026 \\
\hline SRM-981 564 pg & 1.00093 & .021 & 5.2 & 63 & 0.002 & 564 & 4 & 0.05900 & 0.071 & 0.91479 & 0.029 & 2.16765 & 0.068 \\
\hline SRM-981 1874 pg & 1.00091 & 0.018 & 3.6 & 51 & 0.002 & 1874 & 2 & 0.05901 & 0.050 & 0.91483 & 0.017 & 2.16807 & 0.032 \\
\hline Bruderheim & 1.00096 & 0.030 & 2.8 & 55 & 0.293 & 1467 & 2 & 0.04874 & 0.094 & 0.82400 & 0.037 & 1.96001 & 0.086 \\
\hline BCR-1 & 1.00100 & 0.023 & 3.2 & 128 & 15.569 & 2958 & 4 & 0.05312 & 0.044 & 0.83080 & 0.024 & 2.05765 & 0.081 \\
\hline
\end{tabular}

982 agree with most recently published values within $0.02 \%$. We note that analyses of very small loads, such as are reported here, are not suitable for establishing accurate values for standards, and the comparison in Table S5 serves only to demonstrate agreement with the published values obtained from much larger quantities of $\mathrm{Pb}$ per analysis.

An additional complication in comparing our data with the published values is the lack of consistency in the way in which the errors are reported. Our preferred way is to report two errors for each set of standard measurements: standard deviation for evaluation of reproducibility of analyses, and standard error, or, preferably, weighted average with the $95 \%$ confidence error of the mean, for evaluation of the accuracy of the mean value, and for comparing absolute values between studies. In this paper, standard deviations $(2 \sigma)$ are reported in the Tables 2 and S2, and weighted means with $95 \%$ confidence errors of the mean, in the Tables S4 and S5. For some of the published standard data sets, only standard deviations of the population are reported. The published data in Table S5 are presented "as is", without error recalculation: therefore, the difference in reporting errors should be taken into consideration in comparing data sets.

\subsection{Additional factors in $\mathrm{Pb}$ isotope analysis with ${ }^{202} \mathrm{~Pb}-{ }^{205} \mathrm{~Pb}$ DS: $\mathrm{BaPO}_{2}{ }^{+}$interference and anomalous fractionation}

Isotopic ratios corrected for fractionation are expected to be constant, within the error limits of blocks, over the entire temperature range of the run. However, two factors complicate this simple scenario: (1) variable isobaric interferences throughout the run; and (2) non-mass dependent fractionation. The most common interference in $\mathrm{DS} \mathrm{Pb}$ isotopic analysis is from barium phosphate ion $\mathrm{BaPO}_{2}{ }^{+}$(Table 3). The ion beam of $\mathrm{BaPO}_{2}{ }^{+}$was monitored on the mass 201, and a correction was applied to ${ }^{202} \mathrm{~Pb},{ }^{203} \mathrm{~Pb}$ and ${ }^{204} \mathrm{~Pb}$. The contribution of $\mathrm{BaPO}_{2}{ }^{+}$to the ion beam on mass 205 was insignificant in all cases. The $\mathrm{BaPO}_{2}{ }^{+}$ion beam is usually below detection at the beginning of an analysis, but it grows with temperature more rapidly than the $\mathrm{Pb}$ ion beam, and the 201/202 can reach a value of 1 during the second half of a run, when the $\mathrm{Pb}$ ion beam starts to decline. By the end of the analysis of small loads the 201/202 ratio can be as high as 10 and, in rare cases, up to 50 .

The uncertainty involved in the $\mathrm{BaPO}_{2}{ }^{+}$subtraction varies with the 201/202 ratio. A 201/202 value of 1 corresponds to ${ }^{202}\left(\mathrm{BaPO}_{2}\right) /{ }^{202} \mathrm{~Pb}=0.00127$ (average ratio measured in $\mathrm{Pb}$ loads without ${ }^{202} \mathrm{~Pb}$ spike). Assuming a very conservative $10 \%$ uncertainty for $\left.{ }^{201}\left(\mathrm{BaPO}_{2}\right)\right|^{202}\left(\mathrm{BaPO}_{2}\right)$, we get an additional uncertainty estimate of $0.0127 \%$ in the interference corrected ${ }^{202} \mathrm{~Pb} /{ }^{205} \mathrm{~Pb}$, or $0.0032 \%$ per atomic mass unit. This is substantially smaller than the typical analytical errors. An additional error related to $\mathrm{BaPO}_{2}{ }^{+}$subtraction is therefore insignificant for all data with the exception of blocks collected when samples are nearly exhausted. These blocks can be excluded from weighted average calculations. However exclusion or inclusion of these blocks makes very little change in the weighted average values, because of the low intensity of $\mathrm{Pb}$ ion beam and hence larger block value errors, and the weighting by inverse variance involved in the weighted mean calculation effectively discriminates against low-precision data in the populations of blocks with widely variable precision.

The intensity of the $\mathrm{BaPO}_{2}{ }^{+}$beam depends on the amount of barium and phosphorus on the filament. Since $\mathrm{Pb}$ samples are prepared and loaded with $\mathrm{H}_{3} \mathrm{PO}_{4}$, there is always an ample supply of phosphorus on the filament. It is thus important to

Table 3 Abundance ratios of $\mathrm{BaPO}_{2}{ }^{+}$molecular species relative to ${ }^{201} \mathrm{BaPO}_{2}{ }^{+}$, calculated from isotopic composition of $\mathrm{Ba}$ and $\mathrm{O}$

\begin{tabular}{ll}
\hline Mass number & ${ }^{\mathrm{xxx}} \mathrm{BaPO}$ isobar $/{ }^{201} \mathrm{BaPO}_{2}$ isobar \\
\hline 201 & $1.00 \mathrm{E}+00$ \\
202 & $1.39 \mathrm{E}-03$ \\
203 & $4.08 \mathrm{E}-03$ \\
204 & $2.16 \mathrm{E}-06$ \\
205 & $4.12 \mathrm{E}-06$ \\
\hline
\end{tabular}


make sure that the $\mathrm{Pb}$ fraction, and all reagents used in the loading, are free from barium.

Decoupled fractionation of even- and odd-mass isotopes ${ }^{23}$ is another factor that can cause inaccuracy in $\mathrm{Pb}$ isotopic ratios corrected for mass-dependent fractionation using DS. This effect was monitored in all samples by checking the uniformity of normalized ${ }^{205} \mathrm{~Pb} /{ }^{206} \mathrm{~Pb},{ }^{207} \mathrm{~Pb} /{ }^{206} \mathrm{~Pb}$ and ${ }^{208} \mathrm{~Pb} /{ }^{206} \mathrm{~Pb}$ ratios over the temperature range of analysis for a characteristic pattern of odd-even isotope decoupling. We saw a hint of this effect in a few runs of larger standards (e.g., 1000 D, Fig. 1, middle row), but the effect is not nearly as pronounced as in the high-precision analyses of large ( $>50 \mathrm{ng}$ ) $\mathrm{Pb}$ loads. ${ }^{23}$ The decoupled odd-even fractionation can be a serious obstacle to achieving a precision and reproducibility level of 10-20 ppm or better in analyses of large $\mathrm{Pb}$ samples. However, it is unlikely to be a serious problem in the range of sample sizes considered here, for two reasons. First, the magnitude of anomalous odd-even fractionation is usually 3-10 times smaller than the magnitude of mass-dependent fractionation, and would be similar to, or smaller than, the analytical errors for subnanogram $\mathrm{Pb}$ loads. Second, the anomalous odd-even fractionation grows to a substantial magnitude at the end of analyses, after $70-80 \%$ of the total amount of $\mathrm{Pb}$ is evaporated. The ion beam at that stage of analysis usually has low intensity and hence the data are imprecise and are effectively discriminated by inverse variance weighting.

\section{Discussion}

\subsection{Optimizing analysis protocol}

The step heating procedure adopted in this study has several virtues: it allows monitoring of the changes in signal quality over the temperature range, provides good reproducibility of analytical conditions, and can be automated with the available data acquisition software. This procedure proved useful for method development, but it is not necessarily the most efficient in using sample and instrument time. A large portion of the analysis time is wasted in collecting low-precision data at the temperatures outside of the range of optimal emission. It may be better to warm up the sample rapidly to the lower limit of the optimal emission temperature range, and then set the data acquisition program to maintain constant ion beam intensity by occasional increase of the filament temperature, until the sample is exhausted. With such a procedure, it may be possible to use the optimum temperature range more efficiently, and to achieve more uniform data quality between blocks.

Another drawback of the current procedure is the limited possibility of using an ion counting device (secondary electron multiplier or Daly detector). Ion counters are free from thermal noise that limits precision for small signals measured in Faraday cups using amplifiers with feedback resistors. The common problem of ion counters is the instability of gain, which is propagated into the measured isotopic ratios if the ion counter is used as a part of static multi-collector array. This problem can be solved by using a data acquisition sequence with two static multicollector steps, in which a certain isotope is measured in an ion counter in one step, and in a Faraday cup in the other, and the ion counter gain is calculated in each scan. We routinely use such a procedure for measurement of radiogenic $\mathrm{Pb}$ (i.e., $\mathrm{Pb}$ with very low abundance of ${ }^{204} \mathrm{~Pb}$ ) spiked with ${ }^{205} \mathrm{~Pb}$, without DS. The gain, calculated from measurements of the ${ }^{205} \mathrm{~Pb} /{ }^{206} \mathrm{~Pb}$ ratio with ${ }^{205} \mathrm{~Pb}$ in an ion counter and in a Faraday cup, is applied to the measurement of a very small signal of ${ }^{204} \mathrm{~Pb}$ in the ion counter. This procedure is only applicable and efficient if the signal of the reference isotope is large enough for sufficiently precise measurement on a Faraday cup ( $\geq 2-3 \times 10^{-14} \mathrm{~A}$ ), but within the intensity range of the ion counter (about $1 \times 10^{-13} \mathrm{~A}$ for most multipliers). However, a larger ion beam of ${ }^{205} \mathrm{~Pb}$ (at least $\left.2-3 \times 10^{-13} \mathrm{~A}\right)$ is desired in the DS measurements with ${ }^{202} \mathrm{~Pb}-{ }^{205} \mathrm{~Pb}$, in order to take full advantage of internal fractionation correction. Because of these contradictory requirements, the two-step procedure with real time multiplier gain calibration cannot be readily combined with ${ }^{202} \mathrm{~Pb}-{ }^{205} \mathrm{~Pb}$ DS. The conflict may be resolved if a mass spectrometer has greater overlap of dynamic ranges between the low-intensity and high-intensity detectors. This may be achieved, for example, by using $10^{12} \Omega$ feedback resistors for measuring smaller signals in Faraday cups, and by using an analog Daly detector with an intensity upper limit of about $3-4 \times 10^{-13} \mathrm{~A}$ instead of an ion counter.

\subsection{Mass fractionation}

Fractionation factors, calculated from ${ }^{202} \mathrm{~Pb} /{ }^{205} \mathrm{~Pb}$ ratios in sample and standard analyses, are plotted in Fig. 4 against the quantity of $\mathrm{Pb}$.

The fractionation value calculated from ${ }^{202} \mathrm{~Pb} /{ }^{205} \mathrm{~Pb}$ does not vary with sample size, at least for the samples of less than $2 \mathrm{ng}$ analyzed here. There is also no difference in the average fractionation between standards and samples. The average fractionation factors for standards $(0.099 \pm 0.037,2 \mathrm{SD}$, $n=76)$, and samples $(0.095 \pm 0.034,2 \mathrm{SD}, n=86)$ analyzed with DS so far, are identical. The convergence of fractionation

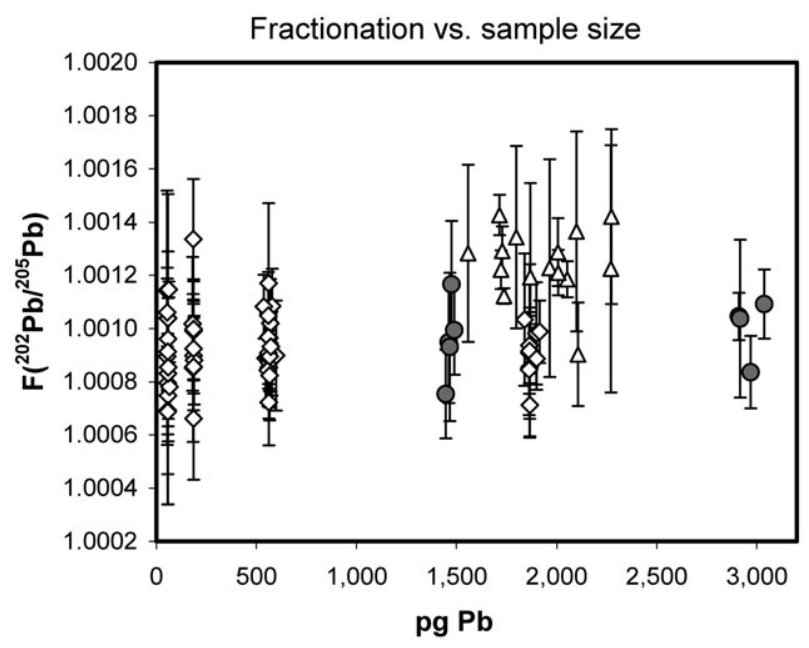

Fig. 4 Fractionation factors per mass unit calculated from the weighted average ${ }^{202} \mathrm{~Pb} /{ }^{205} \mathrm{~Pb}$ ratios, plotted against the sample size. Loads of SRM-981 are shown with open diamonds, spike calibration mixtures (SRM-982 and Mattinson's normal solution) with open triangles, and samples with closed circles. The errors bars are $95 \%$ confidence errors of the mean. 
factors in sample and standard analyses suggests that, with our $\mathrm{Pb}$ separation procedure, the influence of residual matrix on $\mathrm{Pb}$ fractionation is negligible, and that the average fractionation factor determined from standard analyses can be used for external fractionation correction. This observation is at odds with the conclusion of Woodhead et al..$^{7,24,25}$ that a systematic difference in fractionation between $\mathrm{Pb}$ separated from rocks and matrix-free standards is likely. The difference between our observations and those of Woodhead et al. may be related to differences in the efficiency of chemical separation procedures and data acquisition protocols.

The loads of SRM-982 and Mattinson's normal solution, measured during spike calibration, yielded slightly higher fractionation factors than the loads of SRM-981. This is caused by the difference in measuring protocol: the spike calibration loads were measured only until the ion beam intensity was steadily rising in response to heating, whereas SRM-981 loads and $\mathrm{Pb}$ from rocks were heated to nearly complete exhaustion. This example shows that it is important to maintain the same measuring protocol if external normalization is used.

\subsection{Ion yield}

Ion yields plotted versus sample size are shown in Fig. 5. Ion yields for SRM-981 vary between $c a .2$ and 9\%. The values for $\mathrm{Pb}$ separated from rocks are within this range. Lower ion yields, between 0.6 and $2.5 \%$, obtained in spike calibration analyses, are related to the difference in the analysis protocol described in the previous section. The highest ion yield values obtained in this study are similar to the values of Gerstenberger and Haase, ${ }^{1}$ but many analyses yielded lower values. The reason for ion yield variations is currently unknown and is being investigated. Achieving the highest possible sensitivity and reproducible emission conditions will require dedicated studies aimed at understanding the principles and mechanism of ionic emission from molten glasses.

There is a broad tendency of declining ion yields with increasing amount of $\mathrm{Pb}$ on the filament. This tendency is

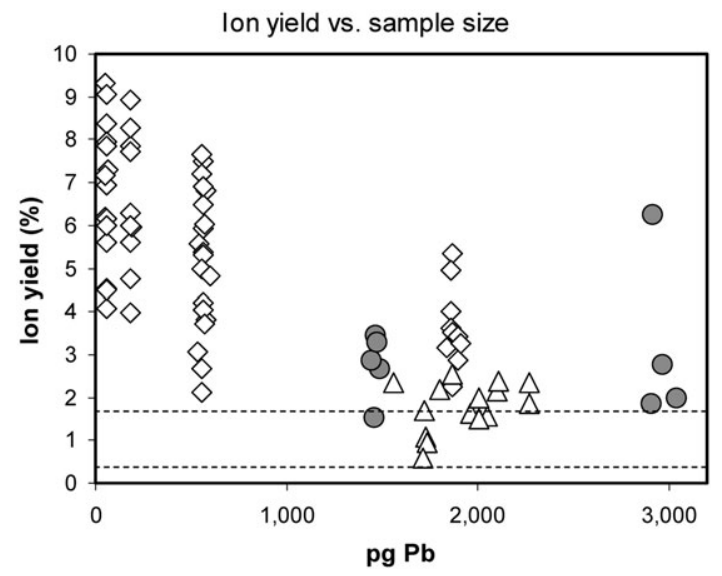

Fig. 5 Ion yields for SRM-981 standard (open diamonds), spike calibration mixtures (open triangles) and samples (filled circles) plotted against the sample size. Broken lines bracket the range of ion yields for $\mathrm{Pb}$ achieved in modern MC-ICP-MS (based on the data in Table $\mathrm{S} 1 \dagger)$. similar to the decline in thorium ionization efficiency with the amount of Th, observed by Edwards et al. ${ }^{26}$ There is, however, a large variation in ion yields at all sample sizes, and we cannot consider this tendency well established until it is confirmed by measurements of much larger samples. Some of the larger standards and samples gave ion yield values of 5-6\%.

Almost all loads, with the exception of a few spike calibration runs, yielded higher ion yields than the highest values achieved in MC-ICP-MS ( $c a .1 .7 \%$ based on the data of Thirlwall: ${ }^{2}$ the values from other studies are between 0.2 and $1.0 \%$, see Table S1). This comparison shows that ion yield in TIMS, although variable, is consistently higher than in MC-ICP-MS. The average TIMS ion yield in this study $(4.6 \%$, Table $\mathrm{S} 2 \dagger)$ is 2.7 times higher than the highest MC-ICP-MS value, whereas the highest TIMS ion yield values are 4-6 times higher than the best MC-ICP-MS values. At the present state of instrumentation and analytical techniques, TIMS is thus the method of choice for precise analysis of small $\mathrm{Pb}$ samples.

\section{4: Data reduction: importance of the weighting procedure}

Various methods are used for calculating the representative values of isotopic ratios from a set of individual integrations or blocks. In conventional arithmetic mean, as well as in nonparametric (e.g. median), calculations we assume that all elements in the set have equal importance. This is clearly not the case for the analysis protocol used in this study, where the ion beam intensity and the within-block precision vary greatly during a typical run. It is necessary therefore to use "a method of computing a kind of arithmetic mean of a set of numbers in which some elements of the set carry more importance (weight) than others". 27 The "weighted average" function of Isoplot Ex, which weights the points by inverse variance, serves this purpose very well. This method assigns greater weights to the data collected at high intensity. The most precise and interference-free data are collected in the middle of the run, where the intensity is the highest. The data collected at the very beginning and the end of the run are more likely to be burdened with problems: organic interferences at the beginning, and $\mathrm{BaPO}_{2}{ }^{+}$interferences and anomalous fractionation at the end of the run. The data quality is thus correlated with intensity and with block error, and the inverse variance weighting works as an objective, and rather efficient, data quality ranking mechanism.

The inverse variance weighting is not necessarily the best method for all sets of isotopic ratios collected from mass spectrometers. The choice of the best method depends on the data distribution. For example, isotopic ratios from solution analyses by MC-ICP-MS are collected at constant intensity, and in that case weighting provides no advantage over conventional or non-parametric calculation. However, weighting may be useful in processing isotopic data collected with transient signals, for example from chromatographic devices.

\subsection{Double spike and sample size limits in isotopic analysis $\mathbf{P b}$}

We can estimate the overall efficiency of our procedure and the ways of improving it by comparing the theoretical limit on precision determined by counting statistics relative to the 


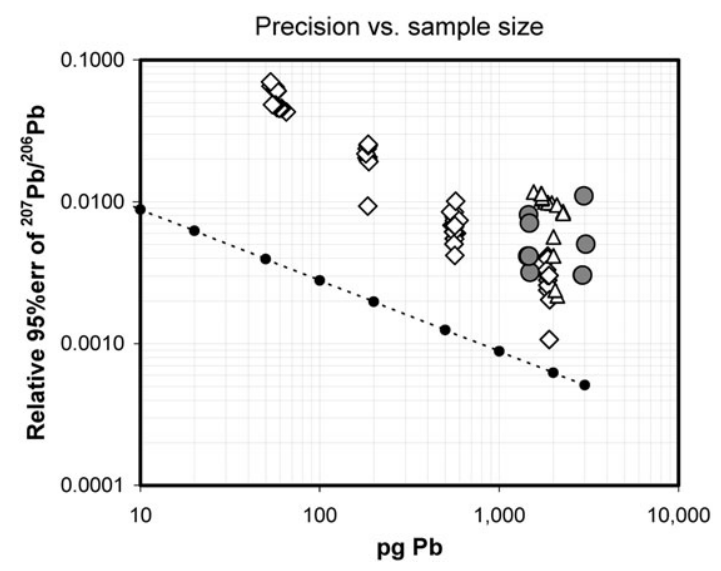

Fig. 6 Precision (95\% confidence error of the weighted average) for DS-normalized SRM-981standard (open diamonds), spike calibration mixtures (open triangles) and samples (closed circles), plotted against the sample size. Dashed curve with small black circles shows theoretical limit on precision based on counting statistics only (from Table S6†).

achieved precision. Assuming an ion yield of $4.6 \%$ (the average value in this study), and $100 \%$ recovery of $\mathrm{Pb}$ from the sample, we should be able to obtain a $2 \sigma$ relative error of $0.01 \%$ for the ${ }^{207} \mathrm{~Pb} /{ }^{206} \mathrm{~Pb}$ ratio for a sample containing $6 \mathrm{pg}$ of ancient radiogenic $\mathrm{Pb}$ with radiogenic ${ }^{207} \mathrm{~Pb} /{ }^{206} \mathrm{~Pb}$ up to 0.62 (ref. 28 and Table S6 $†$ ).

Theoretical precision and achieved precision for ${ }^{207} \mathrm{~Pb} /{ }^{206} \mathrm{~Pb}$ $=0.62$ are compared in Fig. 6 . The most precise analyses from 0.05-0.19 ng loads of SRM-981 are about 10 times less precise than the counting statistics limit, whereas for 0.5-2.0 ng loads the difference between the best-achieved and theoretical precision is $c a$. 3-5 times. This relationship suggests that two groups of factors cause additional errors, some affecting measurements at low ion beam intensity (e.g., electrometer noise and baseline instability, isobaric interferences, and blank) and others independent of intensity (e.g., deviation from the assumed mass fractionation law, and mass independent fractionation). Electrometer noise can be reduced, by a factor of up to 500, by using charge collection (a technique based on using a capacitor in the feedback loop of an electrometer ${ }^{29}$ ). A detailed study of $\mathrm{Pb}$ isotopic fractionation, both mass dependent and anomalous, is in progress (preliminary results are reported in ref. 23).

\subsection{Application of the $\mathrm{Pb} \mathrm{DS}$ procedure in geochronology and cosmochronology}

The technique described here has recently been used for dating early solar system materials: $\mathrm{Ca}-\mathrm{Al}$ rich inclusions and primitive achondrites. ${ }^{30}$ Analyses of multiple fractions containing $0.01-0.3 \mathrm{ng} \mathrm{Pb}$ yielded isochrons with the age error of $0.16-$ 0.33 million years, which was previously unattainable for such small samples.

Future application of our technique in everyday practice of $\mathrm{U}-\mathrm{Pb}$ geochronology would depend on availability of the ${ }^{202} \mathrm{~Pb}-{ }^{205} \mathrm{~Pb}$ spike. We estimate the minimum amount of spike necessary for efficient fractionation correction to be about
$30 \mathrm{pg}$ of each of ${ }^{202} \mathrm{~Pb}$ and ${ }^{205} \mathrm{~Pb}$, therefore one microgram of spike would be sufficient for ca. 30000 analyses of subnanogram $\mathrm{Pb}$ samples, or for a smaller number of nanogram-size $\mathrm{Pb}$ fractions. The limited availability of high-purity ${ }^{202} \mathrm{~Pb}$ and ${ }^{205} \mathrm{~Pb}$ could be an obstacle to wide use of the spike. When the value of increased precision of $\mathrm{U}-\mathrm{Pb}$ dates, provided by the use of the ${ }^{202} \mathrm{~Pb}-{ }^{205} \mathrm{~Pb}$ spike, is demonstrated in a number of case studies of important geological objects, the demand for this spike is likely to increase, and preparation of new batches of high-purity ${ }^{202} \mathrm{~Pb}$ and ${ }^{205} \mathrm{~Pb}$ would become worthwhile.

\section{Summary and conclusions}

The procedure for isotopic analysis of $\mathrm{Pb}$ with fractionation correction using ${ }^{202} \mathrm{~Pb}-{ }^{205} \mathrm{~Pb}$ double spike improves precision and reproducibility of isotopic analyses for samples containing 0.1-3.0 ng Pb compared with the data from the same samples obtained with external normalization. This procedure requires only one analysis per sample to obtain internally fractionation corrected isotopic ratios and $\mathrm{Pb}$ concentrations, is simple, relatively fast, and takes full advantage of the high efficiency of $\mathrm{Pb}$ ionization and low blanks of the TIMS method. The improvement in precision and reproducibility of ${ }^{207} \mathrm{~Pb} /{ }^{206} \mathrm{~Pb}$ ratios using $\mathrm{DS}$ is minor for smaller $\mathrm{Pb}$ loads (less than $0.2 \mathrm{ng}$ ), but becomes more substantial with increasing sample size (3-10 times lower within-run errors, and 2-3 times better reproducibility for $0.5-3.0 \mathrm{ng}$ loads). The procedure is applicable to high-precision $\mathrm{Pb}-\mathrm{Pb}$ and $\mathrm{U}-\mathrm{Pb}$ dating of milligram quantities of meteorites and their components, and single grains of zircon and other U-bearing minerals.

\section{Acknowledgements}

We are grateful to Wolfgang Todt and G. J. Wasserburg who provided high-purity ${ }^{202} \mathrm{~Pb}$ and ${ }^{205} \mathrm{~Pb}$, respectively. Carole Lafontaine, Linda Cataldo and Vicki McNicoll helped at various stages of laboratory work at the GSC. This study was supported by the Geological Survey of Canada, and by a NSERC Discovery grant to Y.A.

\section{References}

1 H. Gerstenberger and G. Haase, Chem. Geol., 1997, 136, 309-312.

2 M. F. Thirlwall, Chem. Geol., 2002, 184, 255-279.

3 J. Baker, D. Peate, T. Waight and C. Meyzen, Chem. Geol., 2004, 211, 275-303.

4 G. D. Kamenov, P. A. Mueller and M. R. Perfit, J. Anal. At. Spectrom., 2004, 19, 1262-1267.

5 M. Elburg, P. Vroon, B. van der Wagt and A. Tchalikian, Chem. Geol., 2005, 223, 196-207.

6 B. Hamelin, G. Manhès, F. Albarède and C. J. Allègre, Geochim. Cosmochim. Acta, 1985, 49, 473-182.

7 J. D. Woodhead and J. M. Hergt, Chem. Geol., 1997, 138, 311-321.

8 S. J. G. Galer, Chem. Geol., 1999, 157, 255-274.

9 M. F. Thirlwall, Chem. Geol., 2000, 163, 299-322.

10 T. Kuritani and E. Nakamura, J. Anal. At. Spectrom., 2003, 18, 1464-1470.

11 W. Compston and V. M. Oversby, J. Geophys. Res., 1969, 74, 4339-4348.

12 N. H. Gale, Chem. Geol., 1970, 6, 305-310.

13 A. W. Hofmann, Earth Planet. Sci. Lett., 1971, 10, 397-402.

14 W. Todt, R. A. Cliff, A. Hanser and A. W. Hofmann, in Earth Processes: Reading the Isotope Code, ed. S. R. Hart and A. Basu, 
Geophysical Monographs, American Geophysical Union, Washington, DC, USA, 1996, vol. 95, pp. 429-437.

15 G. J. Wasserburg, F. Tera, D. A. Papanastassiou and J. C. Huneke, Earth Planet. Sci. Lett., 1977, 35, 294-316.

16 J. C. Roddick, W. D. Loveridge and R. R. Parrish, Chem. Geol., 1987, 66, 111-121.

17 Y. Amelin, A. N. Krot, I. D. Hutcheon and A. A. Ulyanov, Science, 2002, 297, 1678-1683.

18 A. N. Krot, Y. Amelin, P. Cassen and A. Meibom, Nature, 2005, 436, 989-992.

19 Y. Amelin, E. Y. Ritsk and L. N. Neymark, Earth Planet. Sci. Lett., 1997, 148, 299-316.

20 K. R. Ludwig, Isoplot/Ex version 3.00, A Geochronological Toolkit for Microsoft Excel, Berkeley Geochronology Center Special Publication, May 30, 2003. (http://www.bgc.org/Isoplot3 betaManual.pdf).
21 K. R. Ludwig, PBDAT-a computer program for processing $\mathrm{Pb}-$ $\mathrm{U}-\mathrm{Th}$ isotope data, version 1.24. United States Geological Survey Open-File Report 88-542, Revision of June 22, 1993, pp. 1-33.

22 K. Habfast, Int. J. Mass Spectrom., 1998, 176, 133-148.

23 Y. Amelin, D. W. Davis and W. J. Davis, Geochim. Cosmochim. Acta, 2005, 69, A215.

24 J. D. Woodhead, F. Volker and M. T. McCulloch, Analyst, 1995, 120, 35-39.

25 J. D. Woodhead and J. M. Hergt, Geostand. Newsl., 2000, 24, 33-38.

26 R. L. Edwards, J. H. Chen and G. J. Wasserburg, Earth Planet. Sci. Lett., 1987, 81, 175-192.

27 The definition of weighted average is from http://www.mathwords.com.

28 Y. Amelin, Meteoritics Planet. Sci., 2006, 41, 7-17.

29 T. M. Esat, Int. J. Mass Spectrom. Ion Processes, 1995, 148, 159-170.

30 Y. Amelin, M. Wadhwa and G. Lugmair, 37th Lunar and Planetary Science Conference 2006, \#1970. 